\title{
RECOVERING QUASI-REAL OCCLUSION-FREE TEXTURES FOR FACADE MODELS BY EXPLOITING FUSION OF IMAGE AND LASER STREET DATA AND IMAGE INPAINTING
}

\author{
Karim Hammoudi ${ }^{1,2}$ Fadi Dornaika ${ }^{3,4}$ Bahman Soheilian ${ }^{2}$ Bruno Vallet $^{2}$ John McDonald ${ }^{1}$ Nicolas Paparoditis $^{2}$ \\ ${ }^{1}$ Department of Computer Science \\ National University of Ireland Maynooth \\ Maynooth, Co. Kildare, Ireland \\ karim.hammoudi@cs.nuim.ie \\ johnmcd@cs.nuim.ie \\ ${ }^{3}$ Department of Computer Science \\ and Artificial Intelligence \\ University of the Basque Country (UPV/EHU) \\ San Sebastián, Spain \\ fadi_dornaika@ehu.es \\ ${ }^{2}$ Université Paris-Est \\ Institut Géographique National (IGN) \\ Laboratoire MATIS, Saint-Mandé, France \\ bahman.soheilian@ign.fr \\ bruno.vallet@ign.fr \\ nicolas.paparoditis@ign.fr \\ ${ }^{4}$ IKERBASQUE \\ Basque Foundation for Science \\ Bilbao, Spain
}

\begin{abstract}
In this paper we present relevant results for the texturing of $3 D$ urban facade models by exploiting the fusion of terrestrial multi-source data acquired by a Mobile Mapping System (MMS) and image inpainting. Current $3 D$ urban facade models are often textured by using images that contain parts of urban objects that belong to the street. These urban objects represent in this case occlusions since they are located between the acquisition system and the facades. We show the potential use of georeferenced images and 3D point clouds that are acquired at street level by the MMS in generating occlusion-free facade textures. We describe a methodology for reconstructing quasi-real textures of facades that are highly occluded by wide frontal objects.
\end{abstract}

\section{INTRODUCTION AND PROBLEM STATEMENT}

Nowadays, we observe active developments of Mobile Mapping Systems equipped with direct georeferencing devices. These MMSs allow the acquisition of multi-source raw data at street level and provide novel perspectives for enhancing the quality of the terrestrial urban model at wide scale.

In the literature, some research has been conducted for the generation of facade models from terrestrial data. Notably, a classic facade representation is given by georeferenced quadrilaterals (e.g., [1], [2]). Here, our work deals with the generation of facade textures adapted at this frequent level of representation (planar models). More precisely, our interest is focused on the texturing of occluded facades since it constitutes a major challenge for producing complete virtual 3D facade models. This problem is particularly visible in the urban environment given the quantity, the density and the consistency of the street objects.

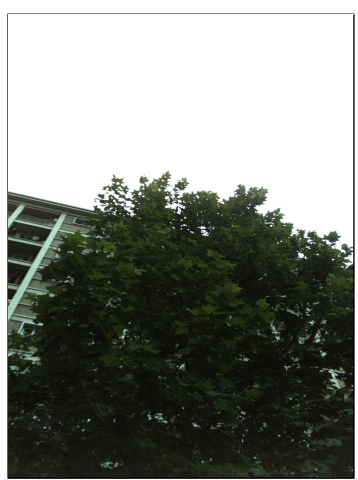

(a) Frontal image of a facade liage. strongly occluded by tree fo-

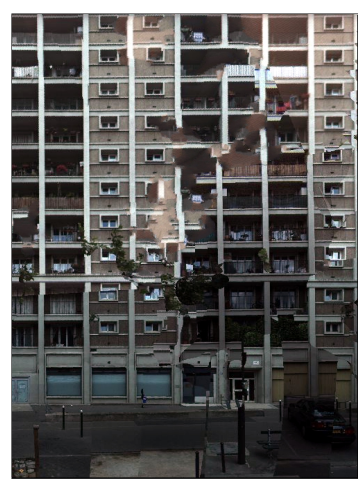

(b) Multiview reconstructed occlusion-free facade texture
Fig. 1. Occlusion-free facade texture reconstructed from a set of multiple facade images acquired by the MMS in displacements along the street and partially completed by image inpainting. Figure 1(a) depicts a worm's-eye view that has been used in the occlusion-free texture computation. This image illustrates the problem complexity in case of direct facade texturing.

Indeed, the facade models often are textured by a mapping with frontal or oblique views of buildings (e.g., [3]). However, these images can be strongly occluded as shown in Figure 1(a). In order to automate the generation of occlusionfree facade texture we must first address the following questions: How can we detect facade occlusions in street images? How can we identify occluded facade image regions and reconstruct a complete occlusion-free texture? (result shown in Figure 1(b)). In the next section, we propose a methodology for answering parts of these open questions. 


\section{PROPOSED APPROACH AND RESULTS}

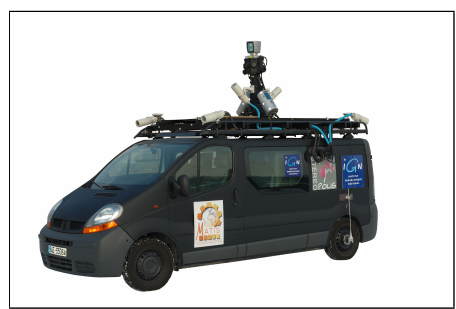

(a) Mobile Mapping System equipped with data positioning instruments and embedded acquisition devices used at street level in on-drive mode.

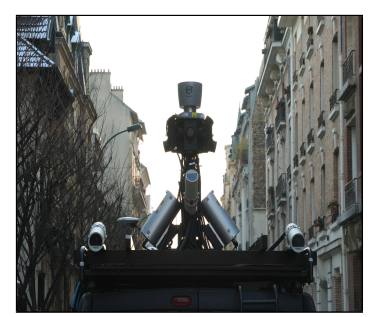

(b) 2D laser range sensor (two cylinders) and omnidirectional optical cameras (octagonal black box) are activated here.

Fig. 2. IGN Mobile Mapping System

The proposed methodology employs data fusion and is in part inspired by [4]. Here, georeferenced terrestrial laser data have been acquired by a MMS in conjunction with a set of calibrated and georeferenced multi-view facade images (see Figure 2). The key idea is to exploit the set of optical images acquired by the MMS in order to produce image transfer that is not affected by occluding objects. Since the view point of the MMS will change due to vehicle motion, one can hope that the occluded parts of a given facade in a set of images will be visible in another set. Inpainting techniques can be applied from the set of images for filling the texture in persistent occluded regions, e.g., non-visible facade regions.

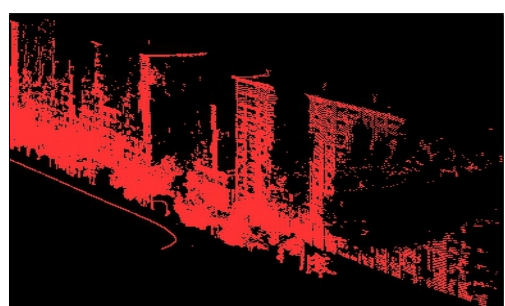

(a) Street point cloud with significant occlusions due to occluding trees.

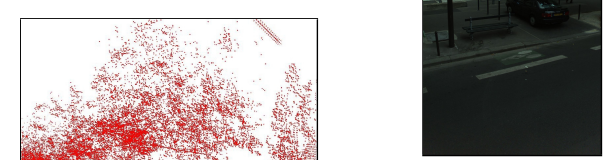

planes are estimated using the method described in [2]. The missing facade laser parts can be qualified as laser shadows and this effect is strongly visible in case of occluding trees since they clearly cause elliptic holes in the facade point cloud (see Figure 3(a)).

Since the facade plane can be estimated as mentioned earlier, the set of 3D points located in a neighbourhood defined by the facade plane and the vehicle trajectory is retained. In certain cases, the retained set of points could also contain points of the ground according to the sensor orientation. The points of ground can be detected and removed by examining their altitude since the bottom delimitation of the facade is assumed to be known. Also, accumulation maps such as described in [2] can be used to identify the presence of the occluding objects. Here, the sensor has been specifically oriented in order to avoid the acquisition of ground points. If the quantity of retained points in the predefined neighbourhood is sufficiently high, then the points are extracted and labelled as occluding points. A result of the occluding point extraction is shown in Figure 3(b).

\subsection{Generating Masks Hiding the Occluding Objects}

(a)

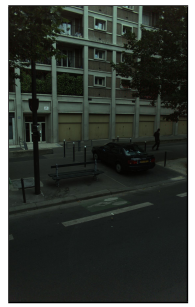

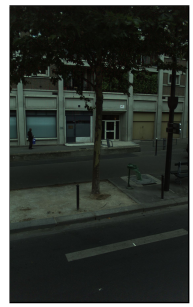

(b)

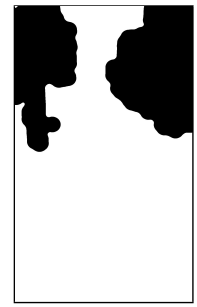

(c)

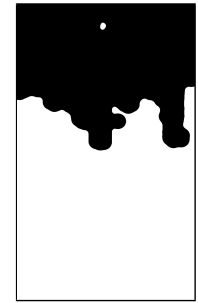

(d)

Fig. 4. (a) and (b) show samples of the multi-view image set. Occluding points previously retained have been projected on. Figures (c) and (d) respectively illustrate the corresponding masks generated by using conventional techniques of mathematical morphology.

points detected in front of the facade planes.

Fig. 3. Sets of points acquired by the MMS in displacements along the street (perspective views). The subset of points in Figure 3(b) has been extracted from the set of points shown in Figure 3(a). The curved line corresponds to the vehicle trajectory.

\subsection{Detecting Occuding Objets of Urban Street Facades}

The occluding objects that are observed in the images of facades can be detected in the laser data at facade level. This is achieved by identifying that the laser beam is intersected by intermediary occluding objects (e.g. vegetation, street lights) located between the point of acquisition and the previously estimated facade planes. As noted in Section 1, the facade

The next task is to detect the occluding objects in the set of optical images. A set of facade images has been associated to each facade according to a visibility criterion based on the size of the image intersection with the projected 3D facade quadrilaterals (which are georeferenced). The detected 3D points that occlude the facade are projected onto the respective set of facade images that have been matched to the facade plane (zoom samples shown Figures 4(a) and 4(b)). We recall that the acquired images are calibrated and all image and laser data are georeferenced. The 3D points are used to generate binary images (masks) with a size identical to the original acquired images $(1920 \times 1080)$. For each of these associated binary images (scattered single points) we apply the morphological operations described below. 


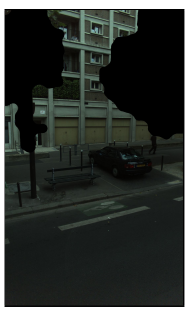

(a)

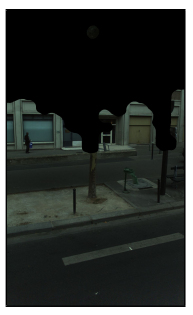

(b)
Fig. 5. (a) and (b) show generated images with occluding objects removed (i.e., image segmentation).

\subsubsection{Amplifying Covering of Occlusions and Filling Gaps}

A morphological dilation is applied onto each projected point in the binary images. The dilation of the binary image $\mathscr{B}$ by the structuring element (i.e., kernel) $K_{d}$ can be defined by (1):

$$
\mathscr{B} \oplus K_{d}=\left\{x \in \mathscr{B} \mid K_{d_{x}} \cap \mathscr{B} \neq \emptyset\right\}
$$

where dilation of $\mathscr{B}$ by $K_{d}$ can then be understood as the set of points $x$ covered by $K_{d}$ when the center of $K_{d}$ (noted $K_{d_{x}}$ ) moves inside objects of $\mathscr{B}$. Here, $K_{d}$ is a large circular Kernel (radius of 50 pixels) that operates onto the totality of the binary image-scattered single points.

\subsubsection{Adjusting the Masks to the Occlusion Size}

A morphological erosion is then applied to the dilated images with a small circular Kernel in order to reduce the amplified size of the masks at the contours. The eroding of the binary image $\mathscr{B}$ by the structuring element (i.e., kernel) $K_{e}$ can be defined by (2):

$$
\mathscr{B} \ominus K_{e}=\left\{x \in \mathscr{B} \mid K_{e_{x}} \subseteq \mathscr{B}\right\}
$$

where the erosion of $\mathscr{B}$ by $K_{e}$ can then be understood as the locus of points reached by the center $x$ of $K_{e}$ (noted $K_{e_{x}}$ ) when $K_{e}$ moves inside $\mathscr{B}$. Here, $K_{e}$ is a small disk (radius of 20 pixels) that operates onto the totality of the previous dilated binary image.

Two examples of the resulting binary masks are shown in Figures 4(c) and 4(d).

\subsubsection{Reducing the Edge Effect Caused by the Kernel Pat- terns}

Finally, a smoothing is applied to the contours of the masks in order to reduce the artificial visual effect caused by the accumulation of circular Kernel patterns. In this way, the rendering of a mosaicing with facade images by subtracting the masks, i.e. reconstructed textures will be not overly affected at the multiple recovered junctions (see results in Figures 5(a) and 5(b)). The benefit of applying this post-processing step can be seen in Figure 1(b). Here although the boundaries due

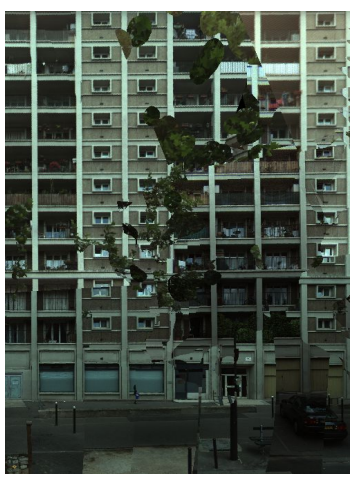

Fig. 6. Real-based texture reconstructed from multiview generated mask images.

to the mask are visible, their appearance is not affected by the kernel's shape.

Then, ortho-image of facade images (with masks) are generated. Finally, a raw mosaicing is achieved by overlapping all the processed facade images excluding the masked regions due to occluding objects (Figure 6$)^{1}$

\subsection{Enhancing Rendering of the Reconstructed Texture}

As it can be seen in Figure 6 it happens that the recovered texture is incomplete in cases of strong occlusion. In this section, we describe the steps we apply to improve the rendering of this reconstructed texture in order to generate a texture more suitable for visualization. In particular, our interest is essentially focused on remaining occluded texture regions.

\subsubsection{Resynthesizing Major Remaining Occluded Parts}

Inpainting techniques can be semi-manually employed in order to process a subset of textures in cases of extreme occlusion. In particular, techniques such as resynthesizing can be directly applied for the removal of external objects in critical parts of the reconstructed facade texture. Here, we have used GIMP $^{2}$ (GNU Image Manipulation Program) with the Resynthesizer $^{3}$ (plug-in developed as a part of [6]). This operation requires delineating targeted regions and filling them by using a powerful transfer method based on the remaining surrounding texture. This practical method is a variant of the best-fit methods described in [7] and [8]. Results of resynthesizing are illustrated in Figures 7(a) and 7(b).

\subsubsection{Adjusting Color Levels of the Resynthesized Texture}

Figure 8(a) presents an unnatural green color tone due to illtuned white balances of the cameras or variation of scene illumination during the acquisition. Figure 8(b) shows a result

\footnotetext{
${ }^{1}$ This part of the presented work was performed at IGN as part of [5].

${ }^{2}$ Available from http://www.gimp.org/

${ }^{3}$ Available from http://www.logarithmic.net/pfh/
} 


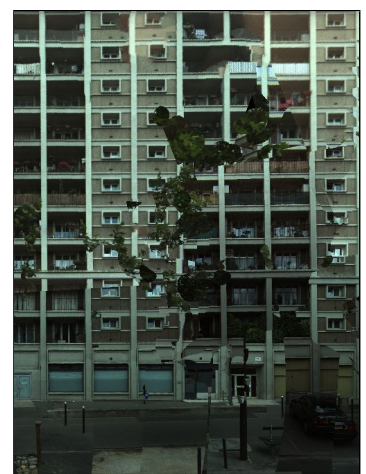

(a) Resynthesized top part initially occluded by vegetation.

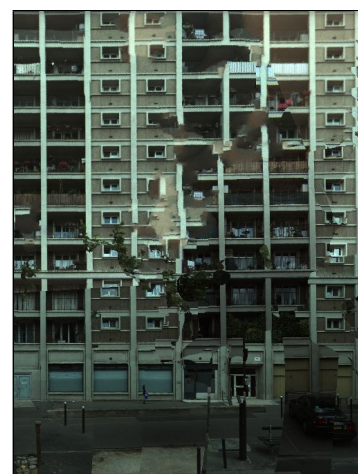

(b) Resynthesized central part initially occluded by vegetation.
Fig. 7. Illustrations of the texture rendering enhancement by progressively applying resynthesizing operations in critical regions from the reconstructed texture shown in Figure 6.

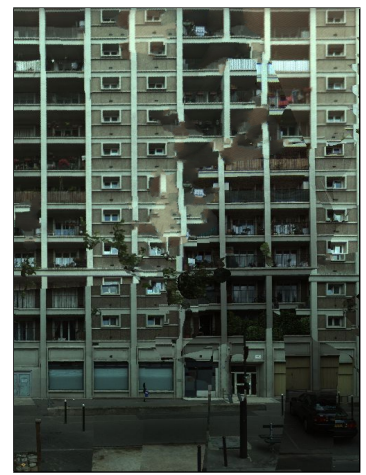

(a) Reconstructed and resynthesized facade texture (Figure 7(b)).

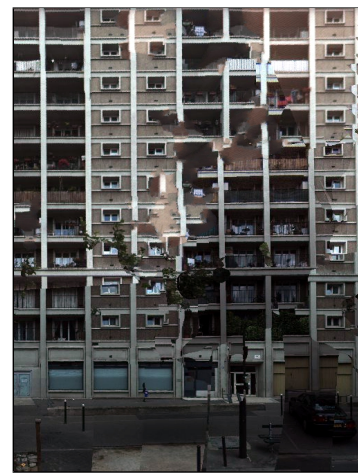

(b) Recovered facade true colors after adjustment.
Fig. 8. Facade texture observed before and after adjustment of the color levels.

of global adjustment of the color levels by applying an Automatic White Balance (AWB) functionality of GIMP onto the Figure 8(a). Facade true colors are recovered with success. This functionality can also be applied manually by picking master colors.

We point out the fact that these semi-manual stages represent minimal interventions and the texture finally produced is quasi-real. Furthermore, we have currently avoided rendering improvements based on the image blurring or image blending in real facade texture parts in spite of remaining imperfections. Indeed, our interest lies in the preservation of the radiometric richness for pixel intensities located in the recovered real facade texture regions (e.g., for image-based postcomputations or for zooming).

\section{CONCLUSION AND FUTURE WORKS}

Initially, the methodology fuses laser raw data and multi-view images and exploits fundamental image processing techniques for masking facade occlusions. Although a raw mosaicing is applied, we observe in Figure 1(b) that the employed technique is able to substantially extend the conventional workflow of occluded texturing (e.g., [3]). The obtained results have been produced from a real set of multi-source urban street data. Furthermore, complex facade textures automatically generated by computer vision techniques are then semimanually inpainted by using some computer graphics techniques. The aesthetic quality of the produced final textures is enhanced for the facade visualization in case of strong occlusions or missing facade regions. Future work will be to improve the mask geometry (occlusion covering) and the texture radiometry (local equalization of the mask images) as well as to optimize the number of images employed in the mosaicing method. Also we intend to apply standard mosaicing algorithms (e.g., [9]) to improve the inter-patch alignment and hence the overall photo-realism of the output. Other investigations can also be led towards automating stages for texture inpainting.

\section{ACKNOWLEDGEMENTS}

The work was in part funded by a Strategic Research Cluster grant (07/SRC/I1168) by Science Foundation Ireland under the National Development Plan. The authors gratefully acknowledge this support.

\section{REFERENCES}

[1] Dragomir Anguelov, Carole Dulong, Daniel Filip, Christian Frueh, Stephane Lafon, Richard Lyon, Abhijit Ogale, Luc Vincent, and Josh Weaver, "Google street view: Capturing the world at street level," Computer, vol. 43, pp. 32-38, 2010.

[2] Karim Hammoudi, Fadi Dornaika, Bahman Soheilian, and Nicolas Paparoditis, "Extracting wire-frame models of street facades from 3D point clouds and the corresponding cadastral map," IAPRS/PCV, vol. A38, pp. 91-96, 2010.

[3] Y.K.A. Tan, L.K. Kwoh, and S.H. Ong, "Large scale texture mapping of building facades," IAPRS, vol. B37, pp. 687-691, 2008.

[4] Sébastien Bénitez, Eloïse Denis, and Caroline Baillard, "Automatic production of occlusion-free rectified facade textures using vehicle-based imagery," IAPRS/PCV, vol. A38, pp. 275-280, 2010.

[5] Karim Hammoudi, "Contributions to the 3D city modeling: 3D polyhedral building model reconstruction from aerial images and 3D facade modeling from terrestrial 3D point cloud and images," Ph.D. thesis, Université Paris-Est, 2011.

[6] Paul Harrison, "Image texture tools," Ph.D. thesis, Monash University, 2005.

[7] T. K. Efros, A. A.; Leung, "Texture synthesis by non-parametric sampling," ICCV, pp. 1033-1038, 1999.

[8] David Donovan Garber, "Computational models for texture analysis and texture synthesis," Ph.D. thesis, University of Southern California, 1981.

[9] Richard Szeliski, Computer Vision: Algorithms and Applications, Springer, 2010. 